\title{
REVIEW
}

\section{Current trends in targeted therapy of leukaemia and lymphoma}

\author{
Josipa Skelin ${ }^{1} \quad$ Mariastefania Antica ${ }^{1^{*}}$
}

Decades of cancer and leukaemia research have provided priceless insight into the molecular mechanisms underlying the development and maintenance of malignancies. The ultimate goal of these findings was, and still is, discovering discriminating factors enabling detection or treatment of tumour cells. An important achievement in this field has been the integration of protein chemistry, fluorescence detectors, nanoparticles, optical devices and computational devolvement integrated in the field of flow cytometry, fluorescence activated cell sorting FACS, data analysis and visualisation. Especially important is the onset of computational data mining tools like T-distributed Stochastic Neighbor Embedding ( $t$ SNE), developed by van der Maatenand Hinton ${ }^{[1]}$ and further continuous progress of the machine learning algorithms for visualization of the huge amount of data produced from single cell FACS or mass cytometry analysis.

This concept has forever changed the way scientists and clinicians approach the search for new therapies, shifting the focus from blanket treatments, such as chemotherapy, to specific targeted therapies which hold the stage today. These therapies have shown a profound impact on the treatment of a variety of human cancers, but none more so than hematopoietic malignancies. Here we summarise the targeted therapies currently being used for the treatment of leukaemias and lymphomas, with a brief overview of those presently being investigated.

Generally, targeted therapies can be divided into two main types: small molecule medicines and monoclonal antibodies. Monoclonal antibodies bind to a specific antigen abundantly expressed on a malignant cell, and induce cell death through either apoptosis, complement-

\footnotetext{
Received: August 6, 2019 Accepted: August 22, 2019 Published: August 23, 2019

Correspondence to: Mariastefania Antica, Division of Molecular Biology, Rudjer Boskovic Institute, Bijenicka 54, 10000 Zagreb Croatia; Email: antica@ irb.hr

${ }^{1}$ Division of Molecular Biology, Rudjer Boskovic Institute, Bijenicka 54, 10000 Zagreb Croatia

Citation: Skelin J and Antica M. Current trends in targeted therapy of leukaemia and lymphoma. Adv Gen Pract Med, 2019, 2(1):16-18.

Copyright: (c) 2019 Mariastefania Antica, et al. This is an open access article distributed under the terms of the Creative Commons Attribution License, which permits unrestricted use, distribution, and reproduction in any medium, provided the original author and source are credited.
}

dependent cytotoxicity or antibody-dependent cellular cytotoxicity. Additionally, the antibody can be linked to a toxic molecule (radioisotope or drug) or to the intracellular part of a $\mathrm{T}$ cell receptor (Chimeric Antigen Receptor T-cell, CAR-T) (CAR-T is not covered by this short presentation, for detailed review see Miliotou and Papadopoulou, 2018) ${ }^{[2]}$ and thus serve as a highly specific drug delivery system.

Naked monoclonal antibodies for the CD20 molecule have been in use for more than 20 years in the treatment of non-Hodgkin lymphoma (NHL) and chronic lymphocytic leukaemia (CLL), with Rituximab was the first monoclonal antibody approved for treatment of human malignancies ${ }^{[3]}$. Ofatumumab emerged a decade later as a more stable antibody with a slower dissociation rate, and has since been approved for the treatment of CLL ${ }^{[4]}$. It remains the only fully human antiCD20 antibody available for treatment. Also Obinutuzumab has been approved for treatment of patients with previously untreated CLL and refractory follicular lymphoma ${ }^{[5]}$. Anti-CD20 antibody has been utilized for precision radiotherapy, as is the case with Ibritumomab tiuxetan which bears a radioactive isotope (yttrium-90 or indium-111) and is used for the treatment of $\mathrm{NHL}^{[6]}$. Similarly, Inotuzumab ozogamicin is a conjugate consisting of an anti-CD22 antibody linked to a cytotoxic agent ozogamicin and it is used primarily for the treatment of acute lymphoblastic leukaemia (ALL) ${ }^{[7]}$. Antibodies for CD33 (Gemtuzumab ozogamicin, for acute myeloid leukaemia (AML)), CD52 (Alemtuzumab, for CLL) and CD30 (Brentuximab vedotin, an antibodydrug conjugate for the treatment of NHL and HL) are also currently approved for use, and many more, such as anti-CD27, anti-CD70 and anti-CD25, are still in various phases of clinical trials ${ }^{[4-10]}$. A promising novel approach to therapeutical monoclonal antibodies is the development of bispecific antibodies which target two antigens simultaneously. Blinatumomab binds CD19 on tumour cells and CD3 on T cells which brings them into close proximity and induces $\mathrm{T}$ cells cytotoxicity ${ }^{[11]}$. Blinatumomab is in current use for subsets of ALL, but there are early results indicating its effectiveness in CLL 
and NHL ${ }^{[12-14]}$. The effectiveness of these therapies depends highly on the treatment regime, dosage and the disease in question. In most cases, monoclonal antibodies are not used as a stand-alone therapy but in various combinations with conventional therapies.

Small molecule medicines in the treatment of hematopoietic malignancies include tyrosine kinase inhibitors, histone deacetylase inhibitors, hypermethylation inhibitors, and proteasome inhibitors. The main targets for tyrosine kinase inhibitors (TKI) are PI3K/AKT, MAPK/ERK, JAK-STAT, STAT5, FLT3 and BTK which have been reported to be aberrantly activated in various acute and chronic leukaemias and lymphomas, typically initiating and maintaining cell proliferation ${ }^{[15,16]}$. The well-known representative of TKIs is the BCR-ABL fusion protein inhibitor used in the treatment of chronic myelogenous leukaemia (CML). Since this initial discovery, a number of inhibitors have been approved for use in the treatment of CML, AML and ALL and more are still in clinical and preclinical trials ${ }^{[17-19]}$.

Histone deacetylases regulate gene expression and, with it, cell proliferation, differentiation and apoptosis. They behave as oncogenes in multiple cancer types, making them ideal therapeutical targets. Histone deacetylase inhibitors (HDI) cause cell death by cell cycle arrest, apoptosis induction or autophagy inhibition but much is unknown of the precise mechanism of action. There are currently four approved pan-HDIs for the treatment of multiple myeloma (Panobinostat) and Tcell lymphoma (Belinostat, Vorinostat and Romidepsin) and several more are in phase II and III of clinical trials ${ }^{[20]}$. All in all, HDIs represent a promising new therapeutic approach, especially when combined with other anti-cancer agents.

Besides histone modifications, transcription is epigenetically regulated by DNA methylation. Aberrant DNA methylation is a common feature of malignancies. In blood cancers, mutations in DNA methylation regulators, such as DNMT3A, TET1/2 and IDH1/2, are commonly found in AML and myelodysplastic syndrome (MDS) in which the hypermethylation patterns can serve to characterise specific subtypes of the disease ${ }^{[21]}$. Therefore, hypermethymation inhibitors represent an interesting therapeutical target. Azacitidin and Decitabine are approved for the treatment of AML and MDS. Patients treated with the inhibitors show an improved overall survival with limited side effects ${ }^{[22,23]}$.

Degradation of cellular proteins is a complex process in which the ubiquitin proteasome pathway represents a central mechanism. Protein degradation is involved in a variety of processes and defects in the ubiquitin proteasome pathway lead to a number of diseases. Inhibiting protein degradation in cells leads to an accumulation of damaged or redundant proteins and, thus, to inhibition of proliferation and induction of apoptosis. Bortesomib was the first proteasome inhibitor to be approved for use in multiple myeloma and mantle cell lymphoma, followed later by carfilzomib and ixazomib, and there is strong evidence indicating that these drugs could also be effective in treatment of AML and ALL ${ }^{[24-26]}$. Even though a number of inhibitors are currently in clinical trials, the three aforementioned are the only presently in use.

With the advancement of available knowledge and technologies, the treatments for hematopoietic malignancies are moving from conventional towards precision therapies. The novel strategies are, mostly, still used with chemotherapy or radiotherapy, but their usefulness is evident in the increase of treatment outcome and patient survival. Targeted therapies are generally well tolerated but have a large range of toxicities, mostly impacting the skin and liver, due to the physiological functions modulated by the drugs. More and more therapeutics are being invented and tested daily, new uses for known drugs are being discovered, and it is clear that the future of cancer treatment lies in targeted therapy.

\section{Acknowledgements}

This work was supported by the funding received from the Croatian science foundation grant IP-11-2013 7140 (Notch in hematopoietic stem cell differentiation and leukaemia development), The Terry Fox Foundation and Croatian League Against Cancer as well as the Ministry of Science and Education of the Republic of Croatia and by the Scientific Centre of Excellence for Reproductive and Regenerative Medicine (Grant Agreement KK01.1.1.01.0008 which is funded by the European Union through the European Regional Development Fund).

\section{References}

[1] van der Maaten LJP and Hinton GE. Visualizing Data Using t-SNE. Journal of Machine Learning Research, 2008, 9: 2579-2605.

[2] Miliotou AN and Papadopoulou LC. CAR T-cell Therapy: A New Era in Cancer Immunotherapy. Current Pharmaceutical Biotechnology, 2018, 19(1): 5-18. https://doi.org/10.2174/1389201019666180418095526

[3] Mclaughlin P, Grillo-López AJ, Link BK, et al. Rituximab chimeric anti-CD20 monoclonal antibody therapy for relapsed indolent lymphoma: half of patients respond to a four-dose treatment program. Journal of Clinical Oncology, 1998, 16(8): 2825-2833.

https://doi.org/10.1200/JCO.1998.16.8.2825

[4] Zhang and Bodi. Ofatumumab. mAbs, 2009, 1(4): 326-331. https://doi.org/10.4161/mabs.1.4.8895 
[5] Owen C and Stewart DA. Obinutuzumab for the treatment of lymphoproliferative disorders. Expert Opinion on Biological Therapy, 2012, 12(3): 343-351. https://doi.org/10.1517/14712598.2012.657622

[6] Grillo-López and Antonio J . Zevalin: the first radioimmunotherapy approved for the treatment of lymphoma. Expert Review of Anticancer Therapy, 2002, 2(5): 485-493. https://doi.org/10.1586/14737140.2.5.485

[7] Kantarjian HM, Deangelo DJ, Stelljes M, et al. Inotuzumab Ozogamicin versus Standard Therapy for Acute Lymphoblastic Leukemia. The New England journal of medicine, 2016, 375(8): 740-753. https://doi.org/10.1056/NEJMoa1509277

[8] Baron $\mathbf{J}$ and Wang ES. Gemtuzumab ozogamicin for the treatment of acutemyeloid leukemia. Expert Review of Clinical Pharmacology, 2018, 11: 549-559. https://doi.org/10.1080/17512433.2018.1478725

[9] Schweighofer CD and Wendtner CM. First-line treatment of chronic lymphocytic leukemia: role of alemtuzumab. Oncotargets \& Therapy, 2010, 3: 53-67. https://doi.org/10.2147/OTT.S3099

[10] Ansell SM. Brentuximab vedotin. Blood, 2014, 124(22): 3197-3200. https://doi.org/10.1182/blood-2014-06-537514

[11] Landau DA, Tausch E, Taylor-Weiner AN, et al. Mutations driving CLL and their evolution in progression and relapse. Nature, 2015, 526: 525-530. https://doi.org/10.1038/nature15395

[12] Wong R, Pepper C, Brennan P, et al. Blinatumomab induces autologous T-cell killing of chronic lymphocytic leukemia cells. Haematologica, 2013, 98(12): 1930-1938. https://doi.org/10.3324/haematol.2012.082248

[13] Mojtaba A, Kum-Ja L, Ashley W, et al. Clinical use of blinatumomab for B-cell acute lymphoblastic leukemia in adults. Therapeutics and Clinical Risk Management, 2016, 12: $1301-1310$. https://doi.org/10.2147/TCRM.S84261

[14] Sanders S and Stewart DA. Targeting non-Hodgkin Lymphoma with Blinatumomab. Expert Opinion on Biological Therapy, 2017, 17: 1013-1017. https://doi.org/10.1080/14712598.2017.1334053

[15] Berenstein R. Class III Receptor Tyrosine Kinases in Acute LeukemiaBiological Functions and Modern Laboratory Analysis. Biomarker insights, 2015, 10: BMI-S22433. https://doi.org/10.4137/BMI.S22433
[16] Drake JM, Lee JK and Witte ON. Clinical targeting of mutated and wild-type protein tyrosine kinases in cancer. Molecular \& Cellular Biology, 2014, 34(10): 1722-1732. https://doi.org/10.1128/MCB.01592-13

[17] Ohanian M, Cortes J, Kantarjian H, et al. Tyrosine kinase inhibitors in acute and chronic leukemias. Expert Opinion on Pharmacotherapy, 2012, 13(7): 927-938. https://doi.org/10.1517/14656566.2012.672974

[18] Man LM, Morris AL and Keng M. New Therapeutic Strategies in Acute Lymphocytic Leukemia. Current Hematologic Malignancy Reports, 2017, 12(3): 197-206. https://doi.org/10.1007/s11899-017-0380-3

[19] Ling Y, Xie Q, Zhang Z, et al. Protein kinase inhibitors for acute leukemia. Biomarker Research, 2018, 6(1): 8 https://doi.org/10.1186/s40364-018-0123-1

[20] Suraweera A, O'Byrne KJ and Richard DJ. Combination Therapy WithHistone Deacetylase Inhibitors (HDACi) for the Treatment of Cancer: Achievingthe Full Therapeutic Potential of HDACi. Frontiers in oncology, 2018, 8: 92. https://doi.org/10.3389/fonc.2018.00092

[21] Guillamot M, Cimmino L and Aifantis I. The Impact of DNA Methylation in Hematopoietic Malignancies. Trends in Cancer, 2016, 2: 70-83. https://doi.org/10.1016/j.trecan.2015.12.006

[22] Fenaux P. Efficacy of azacitidine compared with that of conventional care regimens in the treatment of higher-risk myelodysplastic syndromes : a randomised, open-label, phase III study. Lancet Oncol, 2009, 10: 223-232. https://doi.org/10.1016/S1470-2045(09)70003-8

[23] Kadia TM, Thomas XG, Dmoszynska A, et al. Decitabine improves outcomes in older patients with acute myeloid leukemia and higher blast counts. American Journal of Hematology, 2015, 90(7): E139-E141. https://doi.org/10.1002/ajh.24036

[24] Csizmar CM, Kim DH and Sachs Z. The role of the proteasome in AML. Blood Cancer Journal, 2016, 6(12): e503. https://doi.org/10.1038/bcj.2016.112

[25] Crawford LJ, Walker B and Irvine AE. Proteasome Inhibitors in Cancer Therapy. Journal of Cell Communication and Signaling, 2011, 5(2): 101-110. https://doi.org/10.1007/s12079-011-0121-7

[26] Park JE, Miller Z, Jun Y, et al. Next-generation proteasome inhibitors for cancer therapy. Translational Research, 2018: S1931524418300446. https://doi.org/10.1016/j.trsl.2018.03.002 